\title{
Measurement of hydrogen isotope concentration in erbium oxide coatings
}

\author{
Ryuju Sato ${ }^{a}$, Takumi Chikada ${ }^{a}$, Hiroyuki Matsuzaki ${ }^{a}$, Akihiro Suzuki $^{a}$, Takayuki Terai ${ }^{a}$, \\ Kazuyoshi Sugiyama ${ }^{\mathrm{b}}$, Hans Maier ${ }^{\mathrm{b}}$ \\ ${ }^{a}$ The University of Tokyo, Tokyo, Japan \\ ${ }^{b}$ Max-Planck-Institut für Plasmaphysik, EURATOM Association, Garching, Germany
}

\begin{abstract}
Hydrogen isotope concentrations in erbium oxide coatings fabricated by filtered arc deposition and metalorganic decomposition have been investigated using nuclear reaction analysis and secondary ion mass spectroscopy. It was found that deuterium concentration in the coatings fabricated by filtered arc deposition was 300-500 atomic parts per million, whereas the deuterium concentration in the coating fabricated by metal-organic decomposition was approximately $2.0 \times 10^{4}$ atomic parts per million due to hydrogen trapping by carbon impurities. Deuterium concentrations in the coatings by filtered arc deposition increased with increasing depth in accordance with the increase of grain boundary area density. An in-plane deuterium distribution of the coating by secondary ion mass spectroscopy proved the segregation with a net-like structure, indicating that the deuterium diffused through the grain boundaries.
\end{abstract}

Keywords: Tritium, Permeation, Tritium inventory, Coating, Erbium oxide, Nuclear reaction analysis

\section{Introduction}

Tritium handling has been studied for long period of time in an effort to establish a safe and efficient fuel cycle in fusion reactors. Since candidate structural structure materials generally exhibit high permeability of hydrogen isotopes in the operational temperature range, it is essential to suppress tritium permeation through pipe walls. For many years, ceramic coatings have developed as one method of reducing tritium permeation $[1,2]$.

Erbium oxide $\left(\mathrm{Er}_{2} \mathrm{O}_{3}\right)$ coatings have recently been studied as one of the most promising tritium permeation barrier (TPB) materials [3-7]. Hydrogen isotope permeation experiments on the $\mathrm{Er}_{2} \mathrm{O}_{3}$ coating have been conducted under various conditions to clarify the permeation mechanism. A series of investigations revealed that $\mathrm{Er}_{2} \mathrm{O}_{3}$ coatings fabricated by filtered arc deposition significantly reduce permeation. It has been also reported that a multi-layer structure significantly decreases deuterium permeation by increasing the number of permeation-step sets: solution and diffusion [7]. The hydrogen diffusion behaviors of $\mathrm{Er}_{2} \mathrm{O}_{3}$ coatings have been discussed in several papers, whereas no information on their hydrogen solution behaviors has been provided. Tritium concentration in the coatings has a significant influence not only on the permeation behavior but also on tritium inventory in blanket systems.

In the present research, hydrogen isotope depth profiles of the $\mathrm{Er}_{2} \mathrm{O}_{3}$ coatings after permeation experiments were measured using nuclear reaction analysis (NRA) with tandem accelerators. NRA is a proven method to investigate tritium inventory in diverter materials, such as tungsten [8,9]. Additionally, in-plane deuterium distributions have been examined by nanoscale secondary ion mass spectroscopy (SIMS). Nanoscale SIMS provides two-dimensional (2D) mapping of elements, which has been helpful in determining the diffusion paths of oxygen in ceramics [10]. The goal of the current study is to examine hydrogen isotope concentrations in $\mathrm{Er}_{2} \mathrm{O}_{3}$ coatings fabricated using filtered arc deposition and metal-organic decomposition through these analyses for a better understanding of tritium behavior.

\section{Experimental details}

\subsection{Coating methods}

Reduced activation ferritic/martenstic (RAFM) steel F82H (8Cr-2W, Heat No. 9753 42W-4) was used for a substrate material. Square plates $(25 \mathrm{~mm} \times 25 \mathrm{~mm}, 0.5$ $\mathrm{mm}$ thick) were mirror-polished. $\mathrm{Er}_{2} \mathrm{O}_{3}$ coatings were fabricated by two methods: filterd arc deposition and metal organic decomposition (MOD) as detailed in Refs. $[5,6]$.

For the filtered arc deposition, an arc current applied on an erbium metal cathode $(99.9 \%$ Er) with filtering of the plasma from metal droplets. Oxygen was introduced into the main chamber, forming an $\mathrm{Er}_{2} \mathrm{O}_{3}$ coating. During the deposition RF-induced bias voltages of -150 $\mathrm{V}$ was applied on the sample to enhance crystallinity [11]. $1.4 \mu \mathrm{m}$ thick coatings were deposited on one side or both sides of the substrate.

The coating procedure of the MOD method includes the following steps; (1) the substrate was pulled up from a liquid precursor at a constant speed of $1.2 \mathrm{~mm} \mathrm{~s}^{-1}$, (2) dried in a drying oven at $120{ }^{\circ} \mathrm{C}$ for $15 \mathrm{~min}$., (3) heat treated to crystallize the $\mathrm{Er}_{2} \mathrm{O}_{3}$ coating. The heat treatment conditions were $550-700{ }^{\circ} \mathrm{C}$ for $10 \mathrm{~min}$. under high-purity hydrogen with $0.6 \%$ water vapor. Coatings with thicknesses of less than $100 \mathrm{~nm}$ were fabricated on one side of the substrate. 


\subsection{Hydrogen isotope introduction}

Hydrogen isotopes (protium and deuterium) were introduced into the coated samples at 400, 500, and $600^{\circ} \mathrm{C}$ using the hydrogen isotope permeation apparatus described in Ref.[12]. Two chambers divided by a sample were separately evacuated up to $10^{-6} \mathrm{~Pa}$. Hydrogen isotopes were introduced into one side (called the upstream) at a pressure of $8.00 \times 10^{4} \mathrm{~Pa}$. The partial pressure of the hydrogen isotopes in the other chamber (called the downstream side) was measured by a quadrupole mass spectrometer (QMS). The downstream was continuously pumped out at less than $10^{-4} \mathrm{~Pa}$ during the experiments. The sample coated on one side was mounted with the coated side facing the upstream in order to avoid surface oxidation on the uncoated side of the sample by contamination molecules in the chamber.

The procedure for introducing hydrogen isotopes is describes as follows. First, a hydrogen permeation experiment was performed at $600^{\circ} \mathrm{C}$ for $17 \mathrm{~h}$ on all samples to standardize their microstructure because a phase change of Er2O3 during the first permeation was reported in our previous study [13]. After the permeation test, the upstream chamber was evacuated to release the hydrogen isotopes from the sample. The hydrogen isotopes were then reintroduced into the sample at 400 or $500^{\circ} \mathrm{C}$. When the steady-state permeation flux was achieved, the sample was rapidly cooled down to room temperature at a rate of approximately $15{ }^{\circ} \mathrm{C} \min ^{-1}$ to retain the hydrogen isotopes in the sample while maintaining the upstream pressure. For the hydrogen isotope introduction at $600^{\circ} \mathrm{C}$, the sample was rapidly cooled down after the $17 \mathrm{~h}$ permeation test. Each sample was labeled to indicate the coating method and introduction temperature, such as "Arc-400" and "MOD500." Samples coated on both sides were denoted with "b" before the introduction temperature, such as "Arcb600."

\subsection{Measurement of hydrogen isotope concentration distribution by nuclear reaction analysis}

NRA procedures for protium and deuterium are explained in detail in Refs. $[14,15]$. A proving beam of ${ }^{15} \mathrm{~N}$ or ${ }^{3} \mathrm{He}$ with diameters of approximately $3 \mathrm{~mm}$ and 1 $\mathrm{mm}$, respectively, was injected to the sample. The ion beam reacted with the hydrogen elements and ejected particles or photons. In order to prove the hydrogen depth profiles in the coatings, the incident beam energy was changed from $6.3 \mathrm{MeV}$ to $9.8 \mathrm{MeV}$ for ${ }^{15} \mathrm{~N}$ and from $0.69 \mathrm{MeV}$ to $4.0 \mathrm{MeV}$ for ${ }^{3} \mathrm{He}$.

For the examination of protium concentration, a nuclear reaction of $\mathrm{H}\left({ }^{15} \mathrm{~N}, \gamma \alpha\right){ }^{12} \mathrm{C}$ was performed by a tandem-type Van der Graf accelerator at the Micro Analysis Laboratory, Tandem accelerator (MALT) at the University of Tokyo [11]. The protium concentration was determined via $\gamma$-ray intensity using a semiconductor detector. The $\gamma$-ray intensity was divided by an ion beam current for normalization and converted to protium concentration using a standard sample, Kapton polyimide film. A nuclear reaction with a very narrow resonance width of $1.8 \mathrm{keV}$ occurred at collision energy of $6.385 \mathrm{MeV}-{ }^{15} \mathrm{~N}$ beam against the surface protium.

In order to obtain deuterium profile, ${ }^{3} \mathrm{He}$ was irradiated by a tandem accelerator at Max-Planck-Institut für Plasmaphysik (IPP) Garching, as explained elsewhere [15]. The deuterium concentration was determined using a proton detector via a reaction of $\mathrm{D}\left({ }^{3} \mathrm{He}, \mathrm{p}\right){ }^{4} \mathrm{He}$. The resonance width of this reaction was approximately $500 \mathrm{keV}$. Therefore, the spectra were deconvoluted using the SIMNRA code [16]. The proton intensity was also normalized by ion beam current and converted to deuterium concentration using a standard sample of amorphous deuterated hydrocarbon (a-C:D). Additionally, Rutherford backscattering spectrometry (RBS) was performed at the same time with the equipment at IPP-Garching, which provided the thickness and composition of the $\mathrm{Er}_{2} \mathrm{O}_{3}$ coatings.

\subsection{Deuterium 2D-mapping by nanoscale secondary ion mass spectroscopy}

SIMS was applied to obtain deuterium 2D mappings for Arc-b500 and MOD-500. Each sample was cut into small pieces $\left(7 \times 7 \mathrm{~mm}^{2}\right)$ and coated with gold to suppress the charge-up effect. Mass spectra and depth profiles for protium, deuterium, carbon, and oxygen were measured using the Cameca NanoSIMS50 ion microprobe (Cameca, Courbevoie, France) described in Ref.[10]. $\mathrm{Cs}^{+}$primary ions were irradiated into samples as a tightly focused ion beam with a probe working diameter. The secondary ions were guided through several transfer lenses to the entrance of the mass spectrometer. During the measurement of the depth profiles, the intensities were collected as a function of sputtering time.

\section{Results}

\subsection{Thickness and chemical composition of $\operatorname{Er}_{2} \mathrm{O}_{3}$ coatings}

The coating thickness and Er composition ratio were measured for Arc-400, Arc-b400, Arc-b500, Arc-b600, and MOD-500 by RBS. The Er ratios of the samples fabricated by the filtered arc deposition were $40 \%$ corresponding to the stoichiometric composition of $\mathrm{Er}_{2} \mathrm{O}_{3}$. By contrast, the $\mathrm{Er}$ ratio in MOD-500 showed very low value of approximately $15 \%$.

\subsection{Protium concentration profile}

Fig. 1 shows protium concentration profiles of MOD400, Arc-500, and Arc-600. The protium was detected near the surface of the $\mathrm{Er}_{2} \mathrm{O}_{3}$ coating, and retained by 40 at. $\%$ in MOD-400 and approximately 25 at. $\%$ in Arc-500 nad Arc-600. The surface protium concentration of MOD-400 was 1.5 times larger than those of Arc-500 and Arc-600. However, the inner protium concentration of MOD-400 was 100 times larger than those of Arc-500 and Arc- 600 . 


\subsection{Deuterium concentration profile}

Fig. 2 indicates deuterium profiles of Arc-400 and Arc-500. Since the nuclear reaction of $\mathrm{D}\left({ }^{3} \mathrm{He}, \mathrm{p}\right)^{4} \mathrm{He}$ has the low resolution, the average concentration at each depth was used to determine the depth profile. The

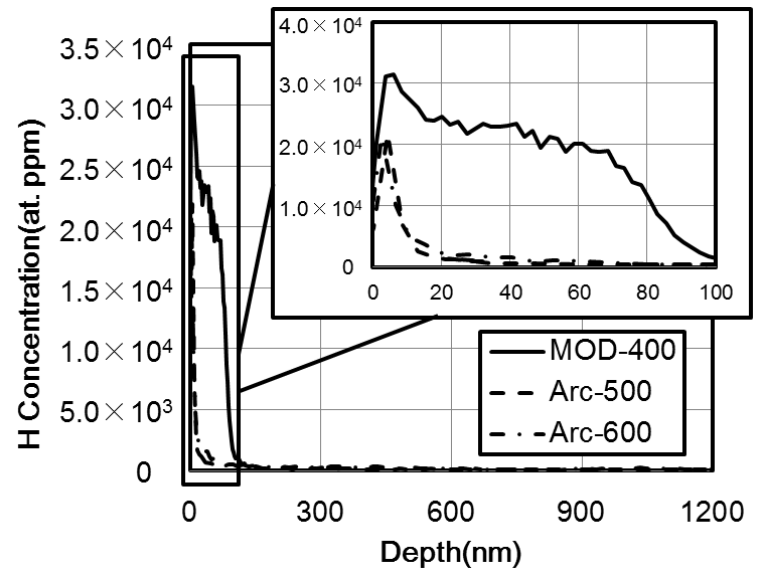

Fig. 1 Protium concentration profiles of MOD-400, Arc-500, and Arc-600. Horizontal axis reoresents the depth from the coating surface, and vertical axis is the protium concentration by atomic parts per million.

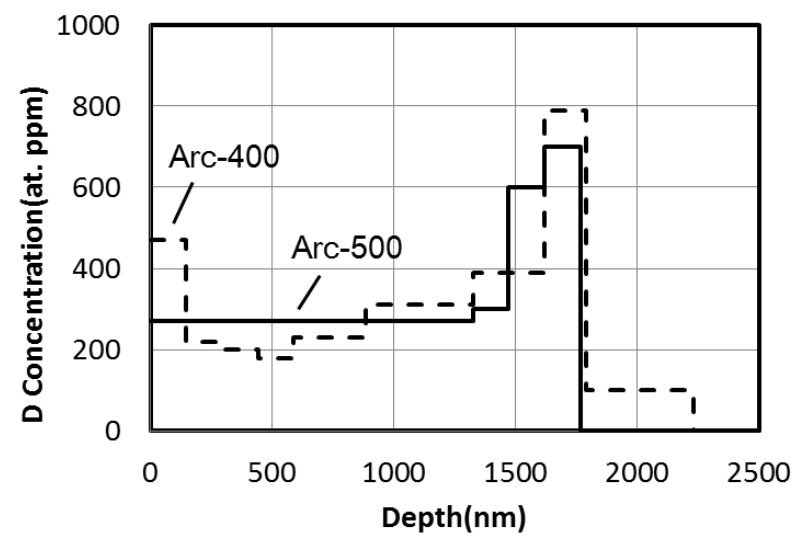

Fig. 2 Deuterium concentration profiles of Arc-400 and Arc500 .

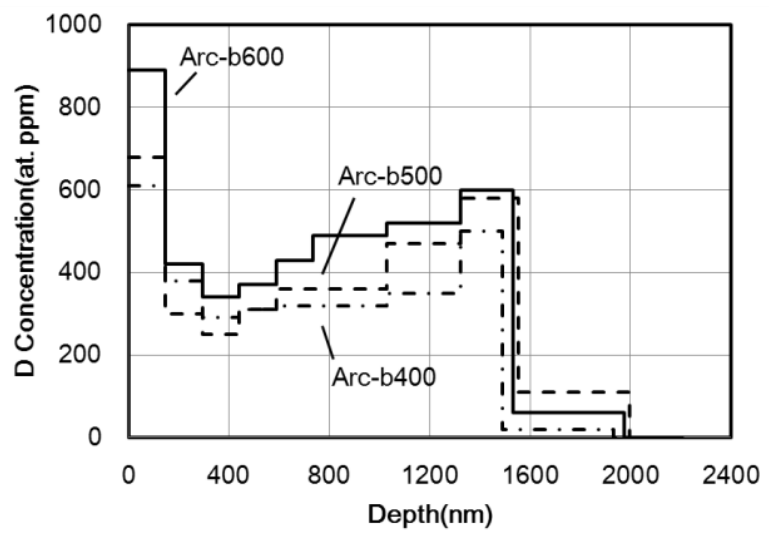

Fig. 3 Deuterium concentration profiles of upstream in Arcb400-600. deuterium profiles in Fig. 2 had peaks near the surfaces, which decreased with depth. However, the deuterium concentrations in the coatings increased with increasing depth and significantly decreased at the substrate. The depth profiles on the upstream side of Arc-b400, Arcb500 and Arc-b600, shown in Fig. 3, showed the same tendency. On the other hand, the coatings facing the downstream side, shown in Fig. 4, show a clear

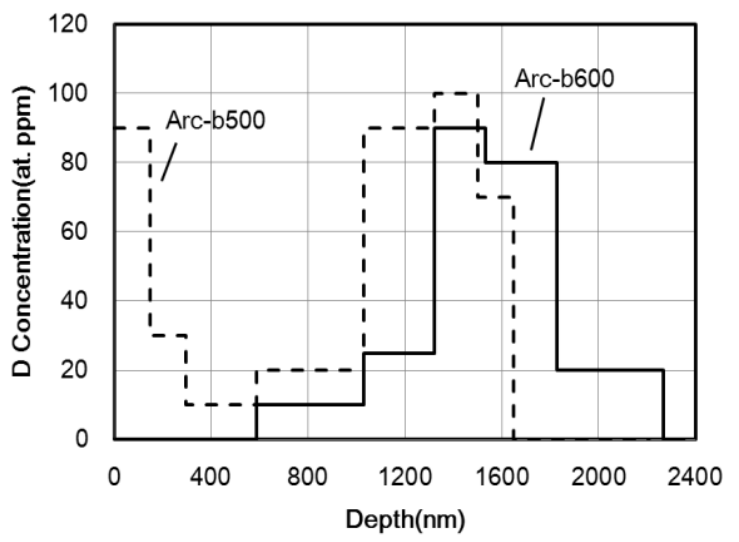

Fig. 4 Deuterium concentration profiles at the downstream of Arc-b500 and Arc-b600.

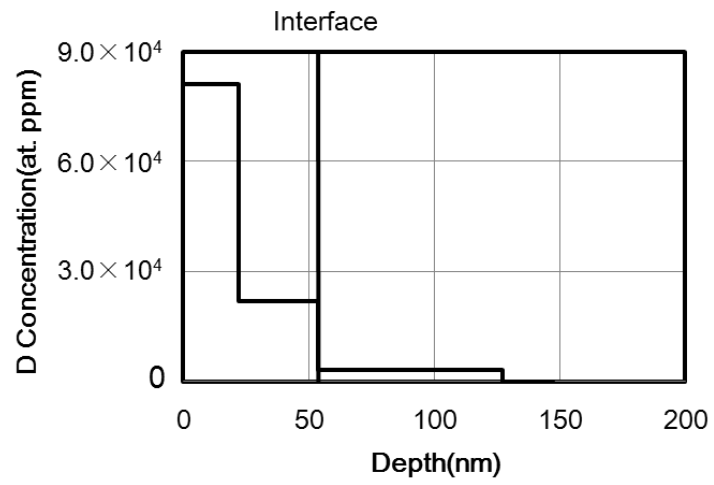

Fig. 5 A deuterium concentration profile of MOD-500. A vertical line at approximately $50-\mathrm{nm}$ depth marks the interface between the $\mathrm{Er}_{2} \mathrm{O}_{3}$ coating and the substrate.

temperature dependence on the deuterium distribution. The distribution on the downstream side of Arc-b500 showed two peaks near the surface and the interface between the coating and substrate, similar to that on the upstream side. However, the deuterium concentration on the downstream side of Arc-b600 was detected only at the interface. Meanwhile, the amount of deuterium in Arc-400 and Arc-b400 was below the detection limit, and therefore the distribution was not available.

The deuterium depth profile of MOD-500 is shown in Fig. 5. The low resolution made it difficult to discuss the deuterium distribution of MOD-500, whose thickness was less than $100 \mathrm{~nm}$. However, it is clear that the MOD coating retained much more deuterium than those fabricated by the filtered arc deposition not only inside but the near surface of the sample. 


\subsection{Deuterium 2D-mapping}

In-plane distributions of deuterium and oxygen in the upstream of Arc-b500 were obtained as Fig. $6 .{ }^{18} \mathrm{O}$ was used for the detection of oxygen because the concentration of ${ }^{16} \mathrm{O}$ was much higher than the detection limit. Although ${ }^{18} \mathrm{O}$ was distributed homogeneously in
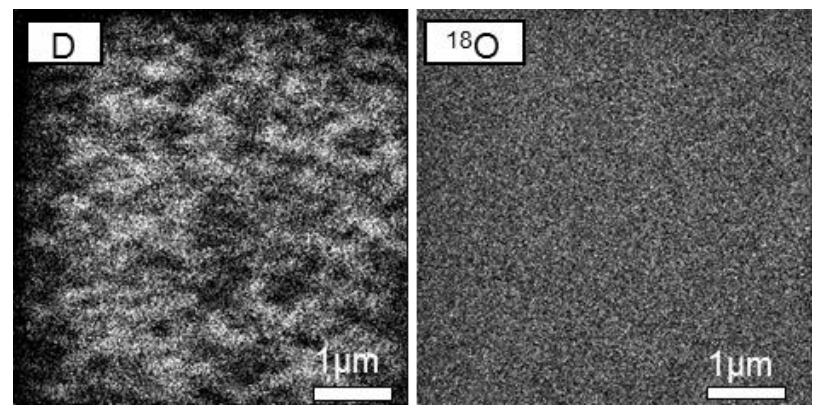

Fig. 6 In-plane distributions of $\mathrm{D}$ and ${ }^{18} \mathrm{O}$ at the upstream of Arc-b500.

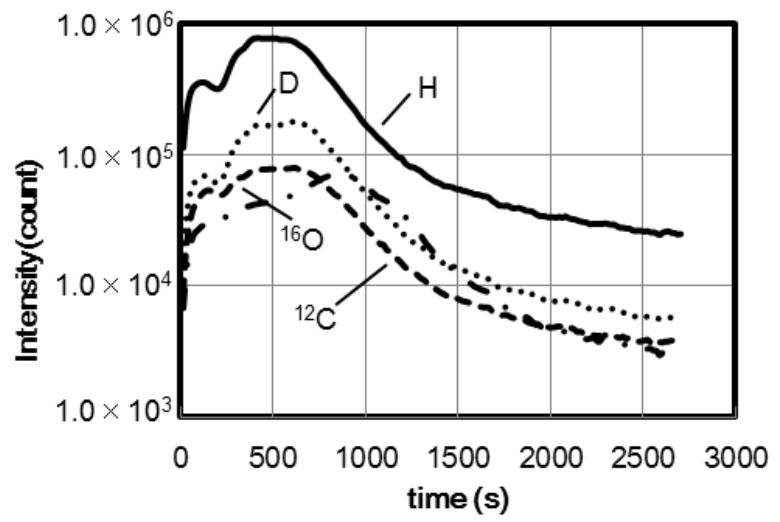

Fig. 7 Depth profiles of protium, deuterium, ${ }^{12} \mathrm{C}$, and ${ }^{16} \mathrm{O}$ in MOD-500. Horizontal axis represents sputtering time, and vertical axis is intensity of each element.
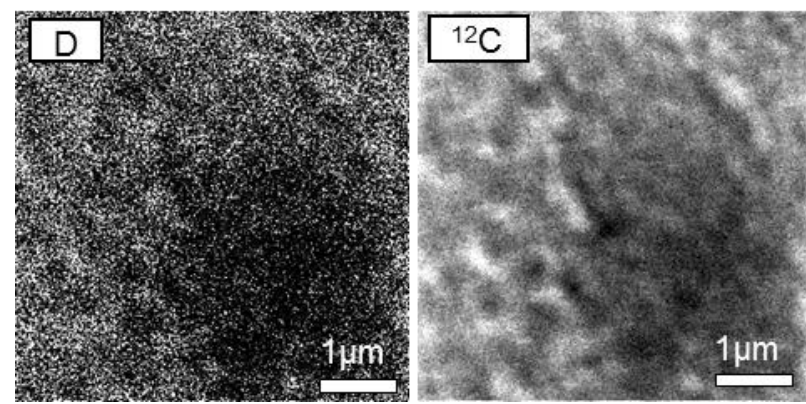

Fig. 8 In-plane distributions of D and ${ }^{12} \mathrm{C}$ in MOD-500.

the sample, deuterium was concentrated in particular places, thus forming a net-like structure. The fineness of the net was approximately $200-500 \mathrm{~nm}$.

Fig. 7 shows depth profiles of protium, deuterium, carbon, and oxygen in MOD-500. At the initial phase, every element increased with the depth. At approximately $700 \mathrm{~s}$ sputtering, protium, deuterium, and carbon started to decrease, and oxygen started to decrease after $1000 \mathrm{~s}$. It is remarkable that the concentrations of protium and deuterium showed a tendency similar to that of carbon. After sputtering for $1000 \mathrm{~s}$, the distribution of each element was measured and the results are shown in Fig. 8. The results indicate that hydrogen isotopes existed locally with carbon but not with oxygen.

\section{Discussion}

In this work, NRA was used to discover the hydrogen adsorption at the coating surface. The protium concentration at the coating surface prepared by filtered arc deposition was 300 times higher than the deuterium concentration, as demonstrated in Fig. 1, Figs. 3 and 4. This large difference was attributed not to the isotopic effect, but to water adsorbed from the air. Hence, it is necessary to eliminate the contribution of water adsorption in order to discuss the protium profiles more precisely.

In the samples fabricated by filtered arc deposition, the local deuterium concentration was obtained at the interface between the coating and substrate as clearly shown in Fig. 3. The results of a previous study indicated that the grain size of the $\mathrm{Er}_{2} \mathrm{O}_{3}$ coating at the interface is smaller than that at the surface, resulting in a larger grain boundary area density at the interface [17]. If hydrogen isotopes diffuse along the grain boundaries, as indicated in Ref. [13], the deuterium concentration at the interface would correspond to the grain boundary density. The deuterium in-plane distribution shown in Fig. 6 proves that deuterium was mainly retained in the grain boundaries, thus forming diffusion paths.

Although the deuterium concentration on the upstream side showed similar behavior at each temperature, the deuterium concentration on the downstream side of samples coated on both sides was clearly influenced by the introduction temperature for the following reasons: (1) the deuterium was not detected at $400{ }^{\circ} \mathrm{C},(2)$ it was retained at both the surface and the coating-substrate interface at $500{ }^{\circ} \mathrm{C}$, and (3) it was found only at the interface at $600{ }^{\circ} \mathrm{C}$. This temperature dependence was attributed to fact that the rates of hydrogen isotope permeation and desorption strongly depend on the temperature. In other words, the deuterium retention was minimal on the downstream side of Arc-b400 due to a small permeation flux, and the deuterium on the surface at $600{ }^{\circ} \mathrm{C}$ disappeared due to the rapid desorption. In order to confirm the latter behavior, thermal desorption spectroscopy will be helpful to determine the temperature at which the hydrogen desorption starts.

The depth profile of each element shown in Fig. 7 revealed that the MOD sample had a complicated structure. According to Fig. 7 and the RBS results, the MOD samples contained many carbon impurities which were thought to come from the metal-organic precursor. It has been reported that MOD samples contain an organic layer on the crystallized $\mathrm{Er}_{2} \mathrm{O}_{3}$ layer [5]. The residual organic layer surely influenced hydrogen 
isotope permeation. Fig. 8 even shows that the deuterium coexisted with carbon, indicating that deuterium formed a chemical bond with carbon. Further investigations on the microstructure of the organic layer and coating are needed for the construction of a permeation model with trap sites.

\section{Conclusion}

Hydrogen isotope concentrations in $\mathrm{Er}_{2} \mathrm{O}_{3}$ coatings fabricated by filtered arc deposition and metal-organic decomposition were investigated using NRA and nanoscale SIMS. The deuterium concentration in the coatings fabricated by filtered arc deposition is 300-500 atomic ppm. On the other hand, the deuterium concentration in MOD coatings is much higher value of approximately $2.0 \times 10^{4}$ atomic parts per million because hydrogen was thought to be trapped by residual carbon.

The deuterium concentration in the coatings by filtered arc deposition increased with increasing depth in accordance with an increase of the grain boundary area density at the interface between the coating and substrate. Additionally, retention of deuterium in grain boundaries was successfully observed by SIMS. These results prove that the deuterium is mainly diffused through in the grain boundaries.

\section{Acknowledgments}

This work was partly supported by Japan Atomic Energy Agency in the framework of the Broader Approach activities, and KAKENHI (25820435), Ministry of Education, Culture, Sports, Science and Technology, Japan.

\section{References}

[1] G. W. Hollenberg, E. P. Simonen, G. Kalinin, A. Terlain, Fusion Eng. Des. 28 (1995) 190-208.

[2] A. Perujo, K. S. Forcey, Fusion Eng. Des. 28 (1995) 252257.

[3] D. Levchuk, S. Levchuk, H. Maier, H. Bolt, and A. Suzuki, J. Nucl. Mater. 367-370 (2007) 1033-1037.

[4] J. Yang, H. Chen, J. Zhang, S. Feng, M. Liu, G. Li, J. Ping, Q. Liu, Y. Yan, Surf. Coat. Technol. 205 (2011) 5497-5501.

[5] T. Chikada, A. Suzuki, T. Tanaka, T. Terai, and T. Muroga, Fusion Eng. Des. 85 (2010) 1537-1541.

[6] T. Chikada, A. Suzuki, T. Terai, T. Muroga, and F. Koch, Fusion Eng. Des., in press (2013) doi: 10.1016/j.jnucmat.2013.03.075.

[7] T. Chikada, A. Suzuki, C. Adelhelm, T. Terai, and T. Muroga, Nucl. Fusion 51 (2011) 063023 (5pp).

[8] K. Sugiyama, M. Mayer, V. Rohde, M. Balden, Th. Dürbeck, A. Herrmann, S. Lindig, A. Wiltner, H. W. Müller, R. Neu and the ASDEX Upgrade team, Nucl. Fusion 50 (2010) 035001 (8pp).
[9] O. V. Ogorodnikova, T. Schwarz-Selinger, K. Sugiyama, T. Dürbeck, and W. Jacob, Phys. Scr. T138 (2009) 014053 (5pp).

[10] I. Sakaguchi, K. Matsumoto, T. Ohgaki, Y. Adachi, K. Watanabe, N. Ohashi, and H. Haneda, J. Ceram. Soc. Japan 118 [5] 362-365

[11] F. Koch, R. Brill, H. Maier, D. Levchuk, A. Suzuki, T. Muroga, H. Bolt, J. Nucl. Mater. 329-333 (2004) 14031406.

[12] T. Chikada, A. Suzuki, Z. Yao, D. Levchuk, H. Maier, T. Terai, T. Muroga, Fusion Eng. Des. 84 (2009) 590-592.

[13] T. Chikada, A. Suzuki, T. Kobayashi, H. Maier, T. Terai, T. Muroga, J. Nucl. Mater. 417 (2011) 1241-1244.

[14] K. Fukutani, M. Tsunoda, Y. Murata, H. Yamashita, K. Komaki, and K. Kobayashi, Surf. Sci. 283 (1993) 447-451.

[15] V. Kh. Alimov, M. Mayer, and J. Roth, Nucl. Inst. Meth. in Phys. Res. B 234 (2005) 169-175.

[16] M. Mayer, SIMNRA User's Guide. Tech. Rep. IPP 9/113, Max-Planck-Institut für Plasmaphysik, Garching, 1997.

[17] C. Adelhelm, T. Pickert, M. Balden, M. Rasinski, T. plocinski, C. Ziebert, F. Koch, H. Maier, Scripta Materialia 61 (2009) 789-792. 